\title{
The Nursing Experience of 215 Cases of Debridement and Suturing Operation of Emergency Trauma in Children
}

\author{
Dehui Shu* \\ The Forth People's Hospital of Zigong City, Zigong, Sichuan 643000, China
}

\begin{abstract}
Objective: To summarize and discuss the nursing of debridement and suturing operation of emergency trauma in children. Methods: Retrospect on the preoperative, intraoperative and postoperative nursing of 125 cases on debridement and suturing operation of emergency trauma in children between January and December 2012. Results: Through a telephone follow-up lasting 3-4 weeks after the operation, there was no complications such as wound infection, wound dehiscence and delayed union among 125 cases of this group, and the wounds were primary healed. Conclusion: Psychological nursing throughout the preoperative, intraoperative and postoperative process, sufficient preoperative preparation, strict aseptic intraoperative operation and close observation, adept nursing cooperation and effective anesthesia can shorten the operation time, reduce the complications and improve the quality of nursing, which are the keys to a successful operation.
\end{abstract}

\author{
KEYWORDS \\ Emergency \\ Debridement \\ Suturing operation \\ Psychological nursing
}

\section{Introduction}

Children are vivacious in nature, they do not have accurate knowledge and judgment on things, and they are also deficient in self-protections and safety awareness, so they can get injured easily in life and study. The children for attending in this department will usually undergo debridement and suturing operation, and they are mostly the only children in the family, so the family members have high expectations on the medical staffs, and are strict on the quality of operation; besides, the risk of operation is higher due to the children's poorer tolerance, cognitive ability, selfadjusting and cooperative ability, which brings many difficulties to the nursing of debridement and suturing, and also makes it more special and important [1]. So sufficient preoperative, intraoperative and postoperative nursing and the enhancement of the quality of operation nursing is the

Copyright @ 2013 Dehui Shu

doi: $10.18686 /$ jn.v2i1.1

Received: February 5, 2013; Accepted: May 14, 2013; Published online: July 13, 2013

This is an open-access article distributed under the terms of the Creative Commons Attribution Unported License (http://creativecommons.org/ licenses/by-nc/4.0/), which permits unrestricted use, distribution, and reproduction in any medium, provided the original work is properly cited.

${ }^{\star}$ Corresponding author: The Forth People's Hospital of Zigong City, Zigong, Sichuan 643000, China. E-mail: shudehui1980@126.com key. 215 children have undergone debridement and suturing operation in the operating room of our emergency outpatient department. Now to report the nursing experiences as follows:

\section{Clinical data}

\subsection{General data}

From January to December in 2012, there were 215 cases of emergency debridement and suturing operation of emergency trauma in children in the operating room of emergency department outpatient in this hospital. There were 141 males and 74 females in this group; age: 3-6 years old: 132 cases, 7-10 years old: 83 cases. Scalp laceration in 120 cases, extremities skin laceration in 70 cases, mandibular skin laceration in 23 cases, penile skin laceration in 1 case, scalp laceration combined with extremities skin laceration in 1 case. The clinical manifestations were mainly skin lacerations, wound pains and bleedings, the patients' crying and restlessness, and the reasons of injuries include fallings, cuts, bites by the animals and car accidents.

\subsection{Operation methods and effects}

All the patients underwent the emergency debridement and suturing operation with local anesthesia, namely local infiltration anesthesia or nerve blocking anesthesia, and used the same anesthetic drugs lidocaine hydrochloride. 
Put the patient with his/her face downwards, first disinfected, anaesthetized, and washed, then proceeded to debridement and suturing, pressure dressing after the operation, and changed the medicine and took out stitches when the patients paid a return visit to the outpatient. There was no wound infection, wound dehiscence, delayed healing or other complications, and the wounds were primary healed.

\section{Nursing}

\subsection{Pre-operative nursing}

\subsubsection{Psychological nursing}

The trauma in the emergency department are mostly accidental, the operation is usually performed in the conscious state, so the psychological guidance is very important, and effective psychological guidance can ensure that the operation can be carried out timely and successfully [2]. When the children leave their parents into a strange environment, the children inevitably will feel terrified and nervous due to the psychological, physiological and other aspects in children and the special nature of the outpatient environment [3]. And the undesirable emotions of nervousness, anxieties and fear from the patient's family members also can affect directly the patient's cooperation, which is very harmful to the safety of operation; debridement and suturing operation requires the recovery of the natural integrity and beauty of the patient's tissue as soon as possible, while the patients' cooperation to a large extent determines the quality of the operation. Therefore the nurses should do the psychological care of patients and their families in a tense and short time, and throughout the whole process of the operation, with a good attitude to cooperate and receive treatment. (1) Treat the patients enthusiastically, inquire actively the reason, the course, the time of the injury, check the injury, whether there is any foreign matter or blood, talk gently, act lightly, use encouraging words to guide the patients in order to eliminate the nervousness and fear, and obtain active cooperation from the patients, enhance the patients' dependence on the operation. If the patients are older with minor injuries, and can tolerate local anesthesia, the nurses should communicate with the patients, encourage the patients to hold out, and admit one of the patients' family members to accompany the patients in the operating room wearing the operating coat when necessary [4]. (2) According to the patients' age characteristics, choose an appropriate way to tell the patients the importance of cooperation to the effects of operation, explain the operation process, how to cooperate, the extent of pains and discomfort, encourage the patients to take the initiative to participate in their own operations, reduce the patients' fear, impulse and antagonistic reactions [5], use other patients or children who have underwent similar operation as modals to illustrate by example the experience of operation after the anesthesia eliminating the pains, dispel the patients' fear about the hospital environment and the operation treatment, and establish the confidence on the operation. (3) Prepare the family members in ideological aspect. Explain the knowledge of operation to the patients' family members, enhance their enthusiasm for cooperative treatment [6], expound explicitly the importance and necessity, the methods and the procedures of debridement and suturing operation, and explain correctly the questions they come up with. According to the different change in patients and their family members' mentality, give psychological guidance targeted in preoperative, intraoperative and postoperative period.

\subsubsection{Preoperative preparations}

(1) The preparation of apparatus. Debridement and suturing package, $1 \%$ lidocaine, $3 \%$ hydrogen peroxide solution, $0.9 \%$ physiological saline, gauze block, $5 \mathrm{~mL}$ syringe and 5.5 trumpet needle, bandage, and according to the wounds conditions and the location of the wounds, prepare different types of needles and sutures. The action should be busy but not chaotic, and arranged in a well-ordered way which makes the patients and their family members feel safe and confident to cooperate with the treatment actively. (2) The preparation of the patients' body posture. Make the patients lie with their faces downwards, one person fixes the injured part with hands, and another person fixed the limbs to avoid the unnecessary restlessness of the patients' limbs, make the wound exposed and the operation visual field, and use the family members' assistance when necessary. (3) The preparation for local anesthesia. The trauma results in the pains around it, and the patients often cry and restless; the mechanical stimulation resulting from the washing of wounds can increase the pains, so the general wound should first be anaesthetized and then washed thoroughly to reduce the pain stimulations [7]. Methods: (1) Sterilization. Sterilize the skin around the wound with iodophor, note that the disinfection should not touch the wound in order to avoid stimulating the wound tissue which can lead to pains. (2) Local anesthesia. In order to relieve the pains of injection, use $5 \mathrm{~mL}$ syringe and No. 7 needle to extract $1 \%$ lidocaine, then replace No. 7 needle with No. 5.5 small needle, then take local infiltration anesthesia or nerve blocking anesthesia on the skin around the wounded part. (3) The preparation of wound. Assist the surgeons in cleaning the wound after making sure that the anesthesia has eliminated the pains, namely first use 3\% hydrogen peroxide solution, then wash with $0.9 \%$ normal saline, the washing should be repeated when the pollution is serious to prevent the anaerobic bacteria infection, for the preparation of debridement and suturing operation in the next step.

\subsection{Intra-operative nursing} 3.2.1. Psychological nursing

(1) The local anesthesia is performed during the operation, so the patients are conscious, have normal thinking, and are mostly accompanied by the family members, so do not 
talk loudly and do not talk about other topics irrelevant to the operation to avoid the undesirable stimulations and reduce the effects of the negative emotions. (2) During the debridement and suturing operation, enjoin the patients and their family member not to looking directly at the wounds or the operation procedures, avoid the patients' eye contact when using the syringe and surgical instruments as far as possible in order to avoid evoking the patients and family member's feeling of nervousness and fear, which can affect the smooth proceedings of the operation, inform the operation process timely, use encouraging and praising words to guide the patients in order to make them cooperate actively during the operation.

\subsubsection{Cooperation with the surgeons during the debri- dement and suturing operation}

Close cooperation of the nurses in the operating room during the operation can not only shorten the operation time, but also enhance the safety of operation, which avoids the occurrence of complications of the operation [8]. (1) Clean the wound thoroughly, including cleaning the foreign matters and blood clots, excising the necrosis slough, stopping bleeding, checking the depth of wounds, whether there is any impair in nerve, tendon, muscle, critical blood vessel, or catalases and etc., and if there are any abnormalities, invite medical staffs in relevant departments to hold a consultation; after trimming the skin around the neat wound, suture the skin smoothly. In order to reduce the postoperative scars, choose 4.0 suture lines in scalp suturing, and choose the 5.0 or 6.0 suture line in the remaining parts. Suture should start from the widest part of the skin tension to the narrowest part gradually, making the skin sutured as a whole. (2) The operation of surgical instruments should be correct and standard, the delivery of instruments should be timely, steady, and accurate. (3) Correct manipulation of operation skills. Take effective anesthesia in the operation, act gently, the manipulation should be adept and fast, and shorten the operation time as far as possible.

\subsubsection{Strict compliance with aseptic manipulation}

Comply with the principle of aseptic manipulation and the rules of operation, supervise each other's performance of sterilized implementation during the operation to avoid cross infection.

\subsubsection{Close observation on the patients' conditions}

In the process of debridement and suturing operation, observe the patients' change in mental situation, consciousness, blood pressure, whether there are symptoms of pale faces, quick pulse, vomiting, sweating and etc. Once the patients have exhibited these symptoms, report to the surgeon immediately to handle appropriately.

\subsubsection{Perform the doctors' advice accurately}

Check the medicine name, dose and prescription, re- tain the surgery ampuoles to the end of the operation for checking.

\subsection{Post-operative nursing 3.3.1. Psychological nursing}

Praise the patients for their performance in front of other patients and parents after the operation, encourage the patients, and eliminate the fear of the second treatment or subsequent visit [9].

\subsubsection{Infection prevention}

(1) Bind up the wounds. The aseptic pressure dressing should be applied according to the injured part after the operation to reduce the chance of bleeding and hematoma, prevent infection and keep the dressing clean, dry. Fix the dressing tight with the adhesive tape to prevent shedding and pollution [10]. If there is pollution or infiltration, inform the medical staffs timely to change. (2) The prescription of medicine. Inject tetanus anti toxin (TAT) or tetanus immune globulin (TIG) in 24 hours after the operation; for the patients with skin laceration resulting from dog's bites, they should be injected into rabies antiserum; apply antibiotics according to the conditions of the wounds appropriately.

\subsubsection{Wounds nursing}

(1) Change fresh dressing for the wounds. Generally, change fresh dressing for the sutured wounds with no drainage in 2-3 days after the operation, for the sutured wound with drainage, change fresh dressing in 24-48 hours after the operation, and later on, change fresh dressing and the wound drainage when it is appropriate. Usually, pull out the drainage in 24-48 hours after the operation according to the draining situations [11]. (2) Taking out stitches. Generally, head: in 5-7 days, limbs: in 10-14 days, trunk limbs: in 7-8 days. (3) Postoperative note. Inform the family members of the location of the wound to avoid physical stimulations such as sweating, wetting and etc. For the younger patients, avoid scratching with hands, lest it loses its functions, making the wound dehiscence [12].

\subsubsection{Regular telephone follow-ups}

Take telephone follow-ups of 71 patients in 4 weeks (70 cases of limbs skin laceration and 1 case of scalp laceration combined with limbs skin laceration) in this group after the operation, and 144 patients in 3 weeks, get acquaintance with the patients' wounds healing timely, and provide health guidance to the patients and family members, making them correct their inappropriate behaviors conscientiously, develop a good habit of life and sanitary, promote the wound-healing as soon as possible [13]. If there exists any abnormality, revisit the doctor. There were no wound infections or laceration during the subsequent visits and telephone follow-ups in this group. 


\section{Nursing experience \\ 4.1. Psychological nursing}

The trauma mostly occurs unexpectedly, the patients and family members are usually at a loss what to do due to the lack of relevant knowledge, and are worried that the postoperative scars will affect the appearance, the manifestations of which are nervousness, anxiety, fear, and are eager to seek medical advice. The nervousness and anxiety can make the muscle tense, which not only affects the patients' cooperation, but also increases their pains, and this is harmful to the smooth proceeding of the operation. The serious negative emotions will not only result in pains, but also bring about a series of negative effects, delaying the healing of wounds, even causing mental disorders and stress ulcers [14]. Therefore, nurses should first actively take psychological care of patients and their family members throughout the various phrases in the preoperative, intraoperative and postoperative period with a good attitude to cooperate and receive treatment, and most of the patients can actively cooperate in the operation through the medical staffs' communication and encouragement.

\subsection{Guarantee the quality of operation nursing and enhance the operation skills}

(1) The caring staffs in the operating room not only should have a high sense of responsibility, but also have abundant clinical experience, and are familiar with professional knowledge in the operating room, continuously strengthen their study in professional work, enhance their professional level, only so can they be familiar with the operation procedures, methods and special requirements when making the debridement and suturing operation. (2) Take various nursing measures to complete preoperative, intraoperative and postoperative nursing enthusiastically, patiently and carefully, and the medical staffs should make the patients and family members obtain the sense of confidence and safety by meticulous working style, adept skill of manipulating, correct technique of operating, making sure that the debridement and suturing operation proceeds smoothly, and ensuring the quality of operation nursing.

\subsection{Health guidance}

(1) Because of the lack of necessary medical knowledge, the family members take inappropriate measures to bind up the wounds and stop the bleeding, which affect the clean sterilization and postoperative healing of the wounds, therefore it is necessary to introduce briefly the prevention of trauma, the basic bind of wounds and the skills of stopping the bleeding to the patients' family members. (2) Postoperative notes. Inform the family members that the dressing should be kept dry and clean to prevent water or other liquid polluting the dressing leading to infection, remind them of the changes in mentality of the patients, and they should pay more attention and caring to the patients, and comfort them because the patients are irritable, crying and restless due to the pains [15]. (3) Guide by telephone follow-ups. Follow-ups by telephone regularly can keep abreast of the state of wound-healing timely, and guide the patients and family members in respective of life and sanitary habits.

All the patients' wounds surface in 215 cases were primary healed, and there was no infection, wound laceration or delayed healing or other complications after all the above nursing measures were taken, which satisfied the family members' operation requirements, and enhanced the efficiency and quality of nursing as well as the family members' degree of satisfaction [16].

In summary, take good psychological care of the patients, encourage the patients to actively cooperate in the operation, and provide psychological guidance to the patients' family members, obtain their positive support and understanding; the operation manipulating skills of the medical staffs, the accurate and effective anesthesia in the operation, the correct application of operation skills, the gentle acts in the operation, and pursuit of "being stable, accurate and fast", all of which are the keys to the debridement and suturing operation. Only by this can enhance the quality of the nursing in the operating room, ensure the quality of medical nursing and medical safety, increase the family members' degree of satisfaction, and improve the hospital's social image.

\section{References}

1. Chen SQ, Wang Y, Li Y. Perioperative nursing of 28 children undergoing finger reconstruction with toe transplantation. Chinese Journal of Nursing. 2013;48(11):973975.

2. Shi SZ. Health Education on patients with emergency debridement suture in 419 cases. Journal of Qiqihaer University of Medicine. 2013;(7):1079-1082.

3. Li X, Peng H, Chen HY. The effect of parents' accompanying on children's anxiety state in pediatric painless colonoscopy examination. Chinese Journal of Behavioral Medicine and Brain Science. 2013;22(8):731-733.

4. Zhou B. Coordination of debridement and suturing for pediatric patients with hand trauma. China Morden Medicine. 2012;19(25):146.

5. Zhang XY, He JY. Influence factors of debridement and suturing in children with maxillofacial trauma under local anesthesia. International Medicine and Health Guidance News. 2013;19(5):655-656.

6. Li LP. Operation Nursing Experience of Minimally Invasive Percutaneous Nephrolithotomy. Medical Innovation of China. 2013;11(1):94-97.

7. Zhou B. Coordination of debridement and suturing for pediatric patients with hand trauma. China Morden Medicine. 2012;19(25):146-148.

8. Fan XY, Guo MQ, Li XL. The Surgical Nursing Cooperation and Experience of Severed Finger Replantation. Medical Innovation of China. 2013;11(2):78-79.

9. Hu R. Application and analysis of four-handed technique 
on children's oral outpatient surgery. Modern Medicine Journal of China. 2013;15(11):73.

10. Zhong B, Yuan JL, Liu LX. Operation cooperation and nursing in pediatric maxillofacial trauma debridement suture. Jilin Medical Journal. 2012;33(14):3094-3095.

11. Zhuang Z, Jiang J. The clinical application of debridement suture. Journal of China Traditional Chinese Medicine Information. 2012;4(4):157.

12. Shi SZ. Health education on patients with emergency debridement suture in 419 cases. Journal of Qiqihaer University of Medicine. 2013;(7):1079-1080.

13. Wu XJ, Chen QJ, Xu QL. The effect of nursing interven- tion on patients' pain, anxiety, depression and stress response in emergency debridement suture. International Journal of Nursing. 2013;32(1):135-139.

14. Cao Y, He YY, SJ. Perioperative nursing of segmentation of finger surgery for children with pointed and (toe) deformity. Chinese Journal of Nursing. 2013;48(10):929-930.

15. Tan WJ. Introduction to technique and nursing experience in pediatric intravenous indwelling needle puncture. Chinese and Foreign Medical Research. 2013;12(4):108-110.

16. Wei YX. Nursing research of emergency debridement suture. China Foreign Medical Treatment. 2013;22(1):157159. 